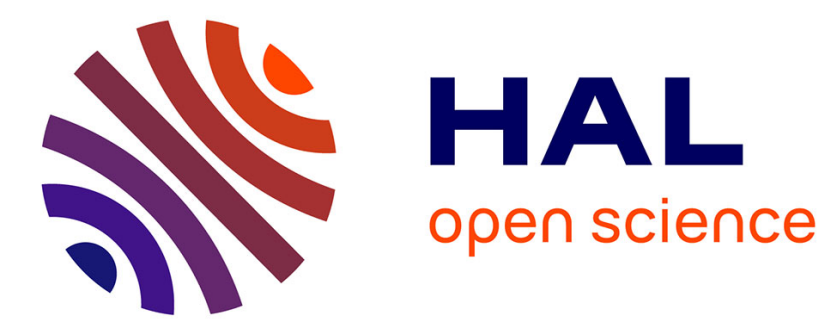

\title{
Credit risk in the pricing and hedging of derivatives
}

Frédéric Abergel

\section{To cite this version:}

Frédéric Abergel. Credit risk in the pricing and hedging of derivatives. 1st Financial Risks International Forum, Paris, Mar 2008, France. hal-00620847

\section{HAL Id: hal-00620847 https://hal.science/hal-00620847}

Submitted on 8 Sep 2011

HAL is a multi-disciplinary open access archive for the deposit and dissemination of scientific research documents, whether they are published or not. The documents may come from teaching and research institutions in France or abroad, or from public or private research centers.
L'archive ouverte pluridisciplinaire HAL, est destinée au dépôt et à la diffusion de documents scientifiques de niveau recherche, publiés ou non, émanant des établissements d'enseignement et de recherche français ou étrangers, des laboratoires publics ou privés. 


\title{
CREDIT RISK IN THE PRICING AND HEDGING OF DERIVATIVES
}

\author{
Frédéric Abergel \\ Chair of Quantitative Finance \\ École Centrale Paris
}

\section{Introduction}

Credit risk is present under various forms in any derivative products. However, the fact is that credit risk is not, in general, priced in the various models commonly used for equity, FX, IR of commodity derivatives. This note is aimed at presenting a few guidelines for the modelling of default risk and spread risk in the context of derivatives, thereby treating any derivative as a genuine credit hybrid. I shall emphasize, using simple examples where the credit risk is described by a reduced-form model, the impact of credit risk in pricing and hedging. It is quite enlightening to revisit the classical strategy of dynamic replication via a "delta" hedge, and understand how it is modified by the credit risk. I shall first use a trivial jump-to-zero model for a risky bond (or a CDS contract) in order to explain in a simplified setting the credit risk effect. Then I will consider a more realistic stochastic default intensity model. Finally, I will briefly present a practical method for the calibration of the joint dynamics. As regards the more theoretical aspects of credit risk modelling, I will rely on the reducedform models such as described in great details in e.g. [BJR] or [SS]. The reason for that choice is that, in capital markets environment, derivative traders are confronted to a host of (more or less) liquid products: stock, vanilla options, variance swaps, CDS, credit index,... and their main objective is the projection of the risks of a given, exotic instrument on existing market instruments. Therefore, one of the main prerequisites for a useful model is that it has good replication properties for the price and the dynamics of the liquid instruments. A typical structural approach for the credit risk, although interesting in itself as it gives rise to an interpretation of the credit event happenstance and therefore, to a better understanding of its governing mechanisms, has the strong disadvantage of making the models and the calibration extremely complicated, with poor performances.

Interestingly enough, there are much fewer references on the hedging of credit risk than there are on its pricing... The pricing by replication with CDS, however natural an extension of the Black-Scholes-Merton theory to the world of credit as it may be, is not commonly used nor exposed in the core of the credit literature. The most relevant references in this direction are [BJR] and [JR]. It is noteworthy that this approach is not well spread in the pure credit derivatives business, where the hedging practices in the industry tend to consider the "spread" risk rather than the actual "default" risk, mostly because of the cost of a full hedging portfolio. However, in the context of credit-contingent options, or in that of counterparty risk management, it is essential to properly measure and eliminate the default risk - which explains why I think the examples below are of interest.

This paper is intended as providing a few examples of how one can explicitly incorporate and manage credit risk into various derivatives. The models presented here, or extensions thereof, 
have been used in a production environment in capital markets for equity and FX derivatives. Section 1 and 2 provide a detailed derivation of the hedging portfolio in a pure jump dynamics for the risky bond, whereas Section 3 deals with the more realistic case of a stochastic spread (stochastic default intensity) model. The short Section 4 tackles the joint calibration problem for a hybrid equity-credit model.

This note is an extension of the talk given by the author during the $1^{\text {st }}$ International Forum on Financial Risks. The author would like to thank the anonymous referee for useful suggestions and comments.

\section{Risky asset contract}

I will first examine the case where a financial institution has entered a contract where an asset $\mathrm{S}$ will be delivered by a counterparty at time $\mathrm{T}$, subject to the counterparty not defaulting prior to T. The contract is then: to deliver $S_{T} 1_{\{\tau>T\}}$ - with standard notations.

\section{a. Uncorrelated case}

By "uncorrelated", I mean that the dynamics of the asset is not affected by a credit event - this is typically the case in a pure counterparty risk hedge. Let's now assume a simple diffusion model for the historical dynamics of the asset

$$
d S_{t}=\sigma S_{t} d W_{t}+\mu S_{t} d t
$$

where $W_{t}$ is a Wiener process, and a pure jump-to-zero model for a risky bond written on the counterparty with the same maturity:

$$
d B_{t}=B_{t-}\left(\lambda d t-d \Pi{ }_{t}\right)
$$

with $\Pi_{t}=1_{\{\tau \leq t\}}$ is the default process.

In this setting, the price of the risky bond is given by $B_{t}=\exp \left(-\int_{0}^{t} \lambda(u) d u\right) 1_{\{\tau>t\}}$ when $\lambda(u)$ is a function of time only, see e.g. [BJR], Section 1.2, and a no-arbitrage argument implies that the drift of the bond must compensate the historical default intensity. However, we will not dwell upon this property here, but rather, describe in detail the hedging argument. We shall use the same convention as in [BJR] and introduce explicitly the dependence in the default time within the function $V_{t}$, thereby setting

$V_{t} \equiv V\left(t, S_{t}, B_{t}, \Pi_{t}\right)$

From now on, we will concentrate on determining the value before default $V\left(t, S_{t}, B_{t}, 0\right)$, and generally assume that the value after default (the recovery) $V\left(t, S_{t}, B_{t}, 1\right)$ is 0 .

Along the classical lines of pricing by replication, a hedge portfolio $P_{t}$ can now be set up making the usual albeit stringent liquidity assumptions on both the asset and the risky bond that will consist at any time of:

1 unit of contract $V_{t}$ 
$\left(-\alpha_{t}\right)$ units of risky bond

$\left(-\beta_{t}\right)$ units of asset.

Given that default has not occurred before the current time t, the change of the value of the portfolio is:

$d P_{t}=d V_{t}-\alpha_{t} d B_{t}-\beta_{t} d S_{t}$

or else, using Itô's formula with jumps in the state variables $(S, B)$ :

$d P_{t}=\frac{\partial V}{\partial S} d S_{t}+\frac{\partial V}{\partial B} \lambda B_{t-} d t+\left(\frac{\partial V}{\partial t}+\frac{1}{2} \sigma^{2} S^{2} \frac{\partial^{2} V}{\partial S^{2}}\right) d t+$

$\left(V\left(S_{t}, 0, t, 1\right)-V\left(S_{t}, B_{t-}, t, 0\right)\right) d \Pi_{t}-\alpha_{t} d B_{t}-\beta_{t} d S_{t}$

The proportions of bond and assets are then determined by:

$\frac{\partial V}{\partial S}=\beta_{t}$

$V\left(S_{t}, 0, t, 1\right)-V\left(S_{t}, B_{t^{-}}, t, 0\right)=-\alpha_{t} B_{t^{-}}$

which allow for a complete elimination of both risks in the dynamics of the portfolio. As this portfolio should now grow at the risk-free (assumed to be zero) rate, the remaining terms in its differential yield the pricing equation for the risky contract:

$$
\frac{\partial V}{\partial t}+\frac{1}{2} \sigma^{2} S^{2} \frac{\partial^{2} V}{\partial S^{2}}+\lambda\left(B \frac{\partial V}{\partial B}+(V(S, 0, t, 1)-V(S, B, t, 0))\right)=0
$$

or, in the simple case where no recovery is expected in the event of a default:

$$
\frac{\partial V}{\partial t}+\frac{1}{2} \sigma^{2} S^{2} \frac{\partial^{2} V}{\partial S^{2}}+\lambda\left(B \frac{\partial V}{\partial B}-V\right)=0
$$

Under the Markovian assumption that the drift is a function of the state variables - in general: $\lambda \equiv \lambda(B, u)$ - the hedging argument yields that the price before default $V$ is the solution to the PDE above, together with the final condition $V(S, B, T, 0)=S$.

For the sake of clarity in this very simple example, I carry out the computations in the "risky asset" contract. One can work out explicitly the solution by separating the variables: a solution is sought under the form $V(S, B, t, 0)=S Q(B, t)$, and the equation for Q is simply:

$$
\begin{aligned}
& \frac{\partial Q}{\partial t}+\lambda\left(B \frac{\partial Q}{\partial B}-Q\right)=0 \\
& Q(B, T)=1
\end{aligned}
$$


Since there is no diffusion of the bond (pure jump), the solution is

$Q(B, t)=\exp \left(-\int_{t}^{T} \lambda(u) d u\right)$

in the case of a deterministic drift $\lambda(u)$ (no dependence on $B$ ) in the dynamics of $B$, as already mentioned at the beginning of this Section. The general solution of the bond pricing equation with an arbitrary final condition $\Psi$ and deterministic drift $\lambda(u)$ :

$\frac{\partial Q}{\partial t}+\lambda\left(B \frac{\partial Q}{\partial B}-Q\right)=0$
$Q(B, T)=\Psi(B)$

is given as

$Q(B, t)=\exp \left(-\int_{t}^{T} \lambda(u) d u\right) \Psi\left(B \exp \left(\int_{t}^{T} \lambda(u) d u\right)\right.$,

which greatly simplifies here since the final condition is a constant.

One recovers the classical result (see e.g. [DS]) that the fair value of the risky contract is equal to market price of the asset* extra discount factor - under the assumption that such a replicating portfolio is constructed. Also of interest is the interpretation of the delta hedge : the hedging strategy is no longer being long one stock (assuming one is short the option), as it would be in a non risky world, but rather, to be long a quantity that goes to one as time to maturity goes to zero. The financing of the continuous rebalancing in stock (buying more and more stock as time to maturity goes to zero) comes through lending money at the risky rate.

\section{b. Correlated case}

I will now look at a more interesting situation: in the case of a default of the counterparty, one can make the assumption that the level of the asset jumps downwards (in the case of a stock, say) or upwards (in the case of an FX rate or a commodity). This situation is typical in the case of systemic risk, it is also relevant for the case of sovereign default. The jump on the asset is governed by the same process as the default of the risky bond:

$$
\begin{aligned}
& d S_{t}=S_{t-}\left(\sigma d W_{t}+\mu d t+(J-1) d \Pi_{t}\right) \\
& d B_{t}=B_{t-}\left(\lambda d t-d \Pi_{t}\right)
\end{aligned}
$$

where $(J-1) S_{t-}$ is the size of the jumps, and (for simplicity) $\mathrm{J}$ is assumed to be constant. Let's write down the pricing and hedging strategy, and derive the pricing equation as above. Assuming again that default has not occurred at time $t$ and setting up a portfolio as in the previous section with

1 unit of contract $V_{t}$ 
$\left(-\alpha_{t}\right)$ units of risky bond

$\left(-\beta_{t}\right)$ units of asset,

the change of the value of the portfolio is:

$d P_{t}=d V_{t}-\alpha_{t} d B_{t}-\beta_{t} d S_{t}$

which now becomes:

$d P_{t}=\frac{\partial V}{\partial S} S_{t-}\left(\sigma d W_{t}+\mu d t\right)+\frac{\partial V}{\partial B} \lambda B_{t-} d t+\left(\frac{\partial V}{\partial t}+\frac{1}{2} \sigma^{2} S_{t-}^{2} \frac{\partial^{2} V}{\partial S^{2}}\right) d t+$

$\left(V\left(J S_{t^{-}}, 0, t, 1\right)-V\left(S_{t^{-}}, B_{t^{-}}, t, 0\right)\right) d \Pi_{t}-\alpha_{t} \lambda B_{t^{-}} d t+\alpha_{t} B_{t^{-}} d \Pi_{t}-\beta_{t} S_{t^{-}}\left(\sigma d W_{t}+\mu d t\right)-\beta_{t}(J-1) S_{t^{-}} d \Pi_{t}$

The proportions of bond and assets are then determined by:

$\frac{\partial V}{\partial S}=\beta_{t}$

$\left(V\left(J S_{t^{-}}, 0, t, 1\right)-V\left(S_{t^{-}}, B_{t^{-}}, t, 0\right)\right)-\beta_{t}(J-1) S_{t^{-}}=-\alpha_{t} B_{t^{-}}$

thereby eliminating both risks in the dynamics of the portfolio. As this portfolio now grows at the risk-free rate, the remaining terms in the differential yield the pricing equation for the risky contract:

$\frac{\partial V}{\partial t}+\frac{1}{2} \sigma^{2} S^{2} \frac{\partial^{2} V}{\partial S^{2}}+\lambda\left(B \frac{\partial V}{\partial B}+(V(J S, 0, t, 1)-V(S, B, t, 0))-(J-1) S \frac{\partial V}{\partial S}\right)=0$

or, in the simple case where no recovery should be expected in the event of a default:

$\frac{\partial V}{\partial t}+\frac{1}{2} \sigma^{2} S^{2} \frac{\partial^{2} V}{\partial S^{2}}+\lambda\left(B \frac{\partial V}{\partial B}-V-(J-1) S \frac{\partial V}{\partial S}\right)=0$.

One now uses the same trick as above to separate the variables: $V(S, B, t, 0)=Q(B, t) W(S, t)$ and the following equations obtain:

$\frac{\partial Q}{\partial t}+\lambda\left(B \frac{\partial Q}{\partial B}-Q\right)=0$
$Q(B, T)=1$

(identical to the previous case)

and

$\frac{\partial W}{\partial t}+\frac{1}{2} \sigma^{2} S^{2} \frac{\partial^{2} W}{\partial S^{2}}-\lambda(J-1) S \frac{\partial W}{\partial S}=0$

$W(S, T)=S$ 
Note the extra convective term coming from the assumption on the dynamics of S. This term obviously degenerates to the case of the previous subsection when there is no jump $(\mathrm{J}=1)$.

It is trivial to check that the solution is $W(S, t)=S e^{-\lambda(J-1)(T-t)}$

in the case of a constant default intensity, and the price of the corresponding hedging strategy - the value at a time $t$ and spot level $\mathrm{S}$ of the risky contract - becomes

$V(S, t)=S e^{-\lambda(J-1)(T-t)} e^{-\lambda(T-t)}=S e^{-\lambda J(T-t)}$

For a term structure of default intensity, one obtains the following: $V(S, t)=S \exp \left(-J \int_{t}^{T} \lambda(u) d u\right)$

\section{Risky option}

\section{a. Uncorrelated case}

Most of what has been stated above remains true if the payoff is of the form $\Phi\left(S_{T}\right) 1_{\{\tau>T\}}$. As a matter of fact, if one tries the separation of variables, one obtains the following equation $\frac{\partial W}{\partial t} Q+\frac{\partial Q}{\partial t} W+\frac{1}{2} \sigma^{2} S^{2} Q \frac{\partial^{2} W}{\partial S^{2}}+\lambda\left(B W \frac{\partial Q}{\partial B}-W Q\right)=0$

where the solution has the form $V=W(S, t) Q(B, t)$. Upon grouping and rearranging the various terms, the following obtains:

$$
Q\left(\frac{\partial W}{\partial t}+\frac{1}{2} \sigma^{2} S^{2} \frac{\partial^{2} W}{\partial S^{2}}-\lambda W\right)=-W\left(\frac{\partial Q}{\partial t}+\lambda B \frac{\partial Q}{\partial B}\right)
$$

or equivalently:

$$
\frac{\frac{\partial W}{\partial t}+\frac{1}{2} \sigma^{2} S^{2} \frac{\partial^{2} W}{\partial S^{2}}-\lambda W}{W}=-\frac{\frac{\partial Q}{\partial t}+\lambda B \frac{\partial Q}{\partial B}}{Q},
$$

and both sides of the equation should then only depend on time: there exists a function $\kappa(t)$ with the property that:

$$
\begin{aligned}
& \frac{\partial W}{\partial t}+\frac{1}{2} \sigma^{2} S^{2} \frac{\partial^{2} W}{\partial S^{2}}-\lambda W=\kappa W, \\
& \frac{\partial Q}{\partial t}+\lambda B \frac{\partial Q}{\partial B}=-\kappa Q
\end{aligned}
$$

It is easy to check that the choice of $k(t)$ is not relevant - different choices will only generate two inverse extra discount factors that will compensate each other - and the obvious choice $\kappa(t)=-\lambda(t)$ leads to the pricing equations : 


$$
\begin{aligned}
& \frac{\partial W}{\partial t}+\frac{1}{2} \sigma^{2} S^{2} \frac{\partial^{2} W}{\partial S^{2}}=0, \\
& \frac{\partial Q}{\partial t}+\lambda B \frac{\partial Q}{\partial B}-\lambda Q=0
\end{aligned},
$$

which shows that the risky price is again equal to non risky price of the option * price of the risky bond in the uncorrelated case.

\section{b. Correlated case}

Again, the fact that there is a jump yields a correction to the risky discount factor: the separation of variables now reads

$$
\frac{\partial W}{\partial t} Q+\frac{\partial Q}{\partial t} W+\frac{1}{2} \sigma^{2} S^{2} Q \frac{\partial^{2} W}{\partial S^{2}}+\lambda\left(B W \frac{\partial Q}{\partial B}-W Q-(J-1) S Q \frac{\partial W}{\partial S}\right)=0,
$$

and for each function Q and W:

$$
\begin{aligned}
& \frac{\partial W}{\partial t}+\frac{1}{2} \sigma^{2} S^{2} \frac{\partial^{2} W}{\partial S^{2}}-\lambda(J-1) S \frac{\partial W}{\partial S}=0, \\
& \frac{\partial Q}{\partial t}+\lambda B \frac{\partial Q}{\partial B}-\lambda Q=0
\end{aligned} .
$$

In order to compare with the option on a non risky asset, solution of

$$
\frac{\partial \widetilde{W}}{\partial t}+\frac{1}{2} \sigma^{2} S^{2} \frac{\partial^{2} \widetilde{W}}{\partial S^{2}}=0
$$

one can use the new state variables $Y=S \exp \left(\int_{t}^{T} \lambda(J-1) d u\right.$ and write the solution to the full equation as in the case of the simple payoff $S_{T} 1_{\{\imath>T\}}$ under the form

$$
V(S, B, t) \equiv Q(B, t) W(S, t)=\exp \left(-\int_{t}^{T} \lambda d u\right) \widetilde{W}\left(S \exp \left(-\int_{t}^{T} \lambda(J-1) d u\right), t\right) .
$$

This formula provides an intuitive interpretation of the pricing formula as discounting with a risky discount factor while correcting the drift in the diffusion for the asset.

\section{c. The case of non zero recovery}

In the case where a recovery is paid at default, the general two-dimensional equation remains identical, but the separation of variables is no longer possible unless one makes the assumption that the recovery is a fraction of the market value before default: $V^{+}=\omega V^{-}$with obvious notations. If this assumption holds, then one still obtains a simple decoupling for the risky option. Otherwise, obtaining the solution requires solving the full 2D PDE.

\section{Spread risk vs. default risk}


In the simple default model of the previous section, the spread risk, that is, exposure to volatility of the spread, was not taken into account. We now address this important question and present a model in which the default intensity is made stochastic in a more explicit manner.

We will now assume that there exist at least two zero-coupon bonds with different maturities that can be used for hedging purposes, thereby eliminating both the jump risk (default risk) and the driving factor in the spread (spread risk). The independent variables are now $S_{t}, \lambda_{t}$ the asset and the stochastic default intensity - so that:

$$
d S_{t}=S_{t-}\left(\sigma d W_{t}^{1}+\mu d t+(J-1) d \Pi{ }_{t}\right)
$$

and $\lambda_{t}$ is a diffusion process

$$
d \lambda_{t}=a d W_{t}^{2}+b d t
$$

(with $\mathrm{a}, \mathrm{b}$ possibly functions of time and intensity level) governing the Cox default process $\Pi_{t}$ . In the sequel, we shall make the assumption that the SDE for the stochastic intensity $\lambda_{t}$ yields nonnegative solutions. This natural and sensible assumption prevents the use of simplistic models, e.g. constant coefficients models, for the intensity. In fact, $\lambda_{t}$ represents the intensity of a Cox process, and has to be nonnegative in order to avoid trivially arbitrageable zero-coupon bonds. Note that the two Wiener processes may be correlated, and that one can specify whether the asset is subject to credit risk $(J \neq 1)$ or not $(J=1)$ when default occurs.

Let's now consider an option with maturity $\mathrm{T}$ conditional to no default before $\mathrm{T}$, and assume that there are two pure credit instruments available, both with maturities larger than $\mathrm{T}$. We will write - similarly to the previous sections:

$V_{t} \equiv V\left(t, S_{t}, \lambda_{t}, H_{t}\right)$

$H_{t}=1_{\{\tau \leq t\}}$

and concentrate on the determination of the value before default $V\left(t, S_{t}, \lambda_{t}, 0\right)$.

Assuming as before that default has not occurred at time $t$ and setting up a hedging portfolio as in the previous section with

1 unit of contract $V_{t}$

$\left(-\alpha_{t}\right)$ units of credit instrument $B_{t}^{1}$

$\left(-\beta_{t}\right)$ units of credit instrument $B_{t}^{2}$

$\left(-\gamma_{t}\right)$ units of asset,

the change of the value of the portfolio can now be written as:

$d P_{t}=d V_{t}-\alpha_{t} d B_{t}^{1}-\beta_{t} d B_{t}^{2}-\gamma_{t} d S_{t}$

which becomes: 


$$
\begin{aligned}
& d P_{t}=\frac{\partial V}{\partial S} S_{t-}\left(\sigma d W_{t}^{1}+\mu d t\right)+\frac{\partial V}{\partial \lambda} d \lambda_{t}+(\Delta V) d \Pi_{t}+\left(\frac{\partial V}{\partial t}+\frac{1}{2} \sigma^{2} S^{2} \frac{\partial^{2} V}{\partial S^{2}}+\frac{1}{2} a^{2} \frac{\partial^{2} V}{\partial \lambda^{2}}+\rho a \sigma S \frac{\partial^{2} V}{\partial \lambda \partial S}\right) d t \\
& -\alpha_{t}\left(\frac{\partial B_{t}^{1}}{\partial \lambda} d \lambda_{t}+\left(\frac{\partial B_{t}^{1}}{\partial t}+\frac{a^{2}}{2} \frac{\partial^{2} B_{t}^{1}}{\partial \lambda^{2}}\right) d t\right)-\beta_{t}\left(\frac{\partial B_{t}^{2}}{\partial \lambda} d \lambda_{t}+\left(\frac{\partial B_{t}^{2}}{\partial t}+\frac{a^{2}}{2} \frac{\partial^{2} B_{t}^{2}}{\partial \lambda^{2}}\right) d t\right) \\
& -\alpha_{t}\left(\Delta B^{1}\right) d \Pi_{t}-\beta_{t}\left(\Delta B^{2}\right) d \Pi_{t}-\gamma_{t} S_{t-}\left(\sigma d W_{t}^{1}+\mu d t\right)-\gamma_{t}(J-1) S_{t-} d \Pi_{t}
\end{aligned}
$$

,where the expression $\Delta X_{t}:=\left(X_{t}-X_{t-}\right)$ stands for the jump of a quantity when default occurs.

The proportions of credit instruments and assets in the hedged portfolio are then determined by solving the following system:

$$
\begin{aligned}
& \frac{\partial V}{\partial S}=\gamma_{t} \\
& \Delta V-\gamma_{t}(J-1) S_{t-}=\alpha_{t} \Delta B^{1}+\beta_{t} \Delta B^{2}, \\
& \frac{\partial V}{\partial \lambda}=\alpha_{t} \frac{\partial B^{1}}{\partial \lambda}+\beta_{t} \frac{\partial B^{2}}{\partial \lambda}
\end{aligned}
$$

thereby eliminating all sources of risks: asset delta, spread risk, default risk.

For the sake of completeness, I derive the pricing equation by introducing the risk premia associated to each risk factor. Similar results may be found in the literature, generally from a different perspective (construction of a martingale measures), see e.g. [S] and also [R].

Now that the hedging strategy is built, the Itô differential of the portfolio becomes:

$d P_{t}=\left(\left(\frac{\partial V}{\partial t}+\frac{1}{2} \sigma^{2} S^{2} \frac{\partial^{2} V}{\partial S^{2}}+\frac{1}{2} a^{2} \frac{\partial^{2} V}{\partial \lambda^{2}}+\rho a \sigma S \frac{\partial^{2} V}{\partial \lambda \partial S}\right)-\alpha_{t}\left(\frac{\partial B_{1}}{\partial t}+\frac{a^{2}}{2} \frac{\partial^{2} B_{1}}{\partial \lambda^{2}}\right)-\beta_{t}\left(\frac{\partial B_{2}}{\partial t}+\frac{a^{2}}{2} \frac{\partial^{2} B_{2}}{\partial \lambda^{2}}\right)\right) d t$ and the portfolio grows at the risk-free (assumed to be zero) rate.

In order to eliminate the weights $\alpha_{t}, \beta_{t}$, we now remark that there are three equations in the two unknowns $\alpha_{t}, \beta_{t}$ :

$$
\begin{aligned}
& \Delta V-\gamma_{t}(J-1) S_{t-}=\alpha_{t} \Delta B^{1}+\beta_{t} \Delta B^{2} \\
& \frac{\partial V}{\partial \lambda}=\alpha_{t} \frac{\partial B^{1}}{\partial \lambda}+\beta_{t} \frac{\partial B^{2}}{\partial \lambda} \\
& \left(\frac{\partial V}{\partial t}+\frac{1}{2} \sigma^{2} S^{2} \frac{\partial^{2} V}{\partial S^{2}}+\frac{1}{2} a^{2} \frac{\partial^{2} V}{\partial \lambda^{2}}+\rho a \sigma S \frac{\partial^{2} V}{\partial \lambda \partial S}\right)=\alpha_{t}\left(\frac{\partial B_{1}}{\partial t}+\frac{a^{2}}{2} \frac{\partial^{2} B_{1}}{\partial \lambda^{2}}\right)+\beta_{t}\left(\frac{\partial B_{2}}{\partial t}+\frac{a^{2}}{2} \frac{\partial^{2} B_{2}}{\partial \lambda^{2}}\right),
\end{aligned}
$$

which states that the three-dimensional matrix 


$$
\left(\begin{array}{cll}
\Delta V-\gamma_{t}(J-1) S_{t-} & \frac{\partial V}{\partial \lambda} & \left(\frac{\partial V}{\partial t}+\frac{1}{2} \sigma^{2} S^{2} \frac{\partial^{2} V}{\partial S^{2}}+\frac{1}{2} a^{2} \frac{\partial^{2} V}{\partial \lambda^{2}}+\rho a \sigma S \frac{\partial^{2} V}{\partial \lambda \partial S}\right) \\
\Delta B^{1} & \frac{\partial B^{1}}{\partial \lambda} & \left(\frac{\partial B_{1}}{\partial t}+\frac{a^{2}}{2} \frac{\partial^{2} B_{1}}{\partial \lambda^{2}}\right) \\
\Delta B^{2} & \frac{\partial B^{2}}{\partial \lambda} & \left(\frac{\partial B_{2}}{\partial t}+\frac{a^{2}}{2} \frac{\partial^{2} B_{2}}{\partial \lambda^{2}}\right)
\end{array}\right)
$$

is of rank strictly less than 3: there exists a zero linear combination of its row vectors with non zero weights. Therefore, its transposed is also of rank strictly less than 3 and there also exists a zero linear combination of its column vectors with non zero weights. Denoting the corresponding coefficient for the first (resp. second) column by $k$ (resp. $q$ ) and assuming that the third coefficient has been rescaled to 1 , the following system of equations obtains easily:

$$
\begin{aligned}
& \frac{\partial B_{1}}{\partial t}+\frac{a^{2}}{2} \frac{\partial^{2} B_{1}}{\partial \lambda^{2}}+k \Delta B^{1}+q \frac{\partial B_{1}}{\partial \lambda}=0 \\
& \frac{\partial B_{2}}{\partial t}+\frac{a^{2}}{2} \frac{\partial^{2} B_{2}}{\partial \lambda^{2}}+k \Delta B^{2}+q \frac{\partial B_{2}}{\partial \lambda}=0 \\
& \frac{\partial V}{\partial t}+\frac{1}{2} \sigma^{2} S^{2} \frac{\partial^{2} V}{\partial S^{2}}+\frac{1}{2} a^{2} \frac{\partial^{2} V}{\partial \lambda^{2}}+\rho a \sigma S \frac{\partial^{2} V}{\partial \lambda \partial S}+k\left(\Delta V-\frac{\partial V}{\partial S}(J-1) S\right)+q \frac{\partial V}{\partial \lambda}=0 .
\end{aligned}
$$

The third equation is the general pricing equation for a default-contingent contingent claim in this model: for any european-style payoff associated to a terminal condition $\Phi_{T}$, the price before default of a default-contingent option $V(S, \lambda, t, 0)$ is given by the solution to the following equation:

$$
\begin{aligned}
& \frac{\partial V}{\partial t}+\frac{1}{2} \sigma^{2} S^{2} \frac{\partial^{2} V}{\partial S^{2}}+\frac{1}{2} a^{2} \frac{\partial^{2} V}{\partial \lambda^{2}}+\rho a \sigma S \frac{\partial^{2} V}{\partial \lambda \partial S}+k\left(\Delta V-\frac{\partial V}{\partial S}(J-1) S\right)+q \frac{\partial V}{\partial \lambda}=0 \\
& V(S, \lambda, T, 0)=\Phi_{T}(S)
\end{aligned}
$$

Let's now examine some consequences of the previous hedging argument.

First of all, a standard argument allows one to assert that risk premia $k, q$ may depend freely on the state variables, but not on the dependent variables such as the bond or option prices. Under the simplifying but natural assumption that the bond prices depend only on time and the default intensity but not on the asset level, $k, q$ must be functions of $t, \lambda$ only. In particular, one can specialize the above pricing equation to obtain the zero-coupon, zerorecovery bond price (no dependence on the asset level) as $B(\lambda, t, 0)$ solution to

$$
\begin{aligned}
& \frac{\partial B}{\partial t}+\frac{a^{2}}{2} \frac{\partial^{2} B}{\partial \lambda^{2}}+q \frac{\partial B}{\partial \lambda}=k B \\
& B(\lambda, T, 0)=1
\end{aligned},
$$

which leads to the classical interpretation of $\mathrm{k}$ as the spread over the risk-free rate, in the sense that there holds:

$$
B(\lambda, t, 0)=E\left(\exp \left(-\int_{t}^{T} k\left(\lambda_{u}, u\right) d u\right) \mid \lambda_{t}=\lambda\right)
$$


where the mathematical expectation is taken under the measure for which the hazard rate follows the diffusion process

$d \lambda_{t}=a d W_{t}^{2}+q d t$.

More generally, the price of an option with european-style payoff $\Phi_{T}$ is valued as $V(S, \lambda, t, 0)=E\left(\exp \left(-\int_{t}^{T} k\left(\lambda_{u}, u\right) d u\right) \Phi_{T} \mid S_{t}=S, \lambda_{t}=\lambda\right)$, where the risk-neutral jumpdiffusion for the asset is given by

$$
d S_{t}=S_{t-}\left(\sigma d W_{t}^{1}+(J-1)\left(d \tilde{\Pi}_{t}-k d t\right)\right)
$$

for a default process $\tilde{\Pi}_{t}$ having the modified intensity $k\left(\lambda_{t}, t\right)$, and the risk-neutral diffusion for the hazard rate is as above:

$d \lambda_{t}=a d W_{t}^{2}+q d t$.

One important question, at least for practical applications, is therefore: what is a suitable choice for $k, q$ ? There are many aspects to take into account, one of them being the numerical tractability of the bond prices. Keeping this in mind, it may be convenient to choose an exponential-affine structure, see [DS] or [DFS], for the special case of a zero coupon, zero recovery bond price. In particular, the volatility of the default intensity should either be a simple term structure, or a square root process. Since the term structure assumption leads to potentially non positive default intensities, it must be rejected.

The choice of an exponential-affine bond structure leads to the following general form for the risk premia:

$k=x(t) \lambda+y(t)$

$q=m(t) \lambda+n(t)$

which in the simplest case $k=\lambda$ reduces to the classical interpretation of the spread. I shall assume in the rest of the paper that the choice $k=\lambda$ has been made.

The bond pricing equation becomes

$\frac{\partial B}{\partial t}+\frac{a^{2}}{2} \frac{\partial^{2} B}{\partial \lambda^{2}}+q \frac{\partial B}{\partial \lambda}=\lambda B$

and the general pricing equation for a credit contingent option on the asset now reads:

$\frac{\partial V}{\partial t}+\frac{1}{2} \sigma^{2} S^{2} \frac{\partial^{2} V}{\partial S^{2}}+\frac{1}{2} a^{2} \frac{\partial^{2} V}{\partial \lambda^{2}}+\rho a \sigma S \frac{\partial^{2} V}{\partial \lambda \partial S}+q \frac{\partial V}{\partial \lambda}+\lambda\left(\Delta V-\frac{\partial V}{\partial S}(J-1) S\right)$.

In the fully correlated case $\mathrm{J}=0$, the pricing equation is:

$$
\frac{\partial V}{\partial t}+\frac{1}{2} \sigma^{2} S^{2} \frac{\partial^{2} V}{\partial S^{2}}+\frac{1}{2} a^{2} \frac{\partial^{2} V}{\partial \lambda^{2}}+\rho a \sigma S \frac{\partial^{2} V}{\partial \lambda \partial S}+q \frac{\partial V}{\partial \lambda}+\lambda S \frac{\partial V}{\partial S}-\lambda V=0 .
$$

Note that, if the option is not credit contingent (standard european option with terminal payoff $\Phi_{T}$ ), but if however a credit event implies a jump on the asset, one obtains the general jumpdiffusion pricing equation: 


$$
\frac{\partial V}{\partial t}+\frac{\sigma^{2}}{2} S^{2} \frac{\partial^{2} V}{\partial S^{2}}+\frac{a^{2}}{2} \frac{\partial^{2} V}{\partial \lambda^{2}}+\rho a \sigma S \frac{\partial^{2} V}{\partial S \partial \lambda}+q \frac{\partial V}{\partial \lambda}+\lambda\left(V(J S)-V-(J-1) S \frac{\partial V}{\partial S}\right)=0 .
$$

It is straightforward to check the consistency of the various equations above: martingale property for the asset price in the fully correlated or the jump-diffusion case, option price depending on the asset only if $\mathrm{J}=1 \ldots$

Remark: one can briefly address the issue of solving the pricing equation for a credit contingent claim. In general, one cannot expect to separate the variables. A natural exception is that of a credit contingent option on a non risky asset $(\mathrm{J}=1)$ with zero brownian correlation, in which case the same formula as in Section 2.b holds. Also true is a similar result using separation of variables for a risky stock contract, as in this case there exists a particular solution of the form $V(S, \lambda, t, 0)=Q(\lambda, t) S$, where $Q(\lambda, t)$ is a solution to the equation below:

$\frac{\partial Q}{\partial t}+\frac{1}{2} a^{2} \frac{\partial^{2} Q}{\partial \lambda^{2}}+\rho a \sigma \frac{\partial Q}{\partial \lambda}+q \frac{\partial Q}{\partial \lambda}-\lambda J Q=0$

with terminal condition $\mathrm{Q}=1$ at maturity. This equation degenerates to the case of Section $1 . \mathrm{b}$ when the default intensity is deterministic.

\section{Calibration of a stochastic "jump to default" case}

In this short section, I tackle the issue of calibration. First and foremost, the credit part of the calibration process is simple in the case of an exponential-affine bond structure, as explained in the previous section: one must first specify the volatility coefficient of the CIR process - a volatility which, in the absence of a liquid option market on CDS, should be determined from time series of spread levels - and then, calibrate via forward induction a term structure for the drift onto the curve of the CDS prices. Assuming that this part is already performed, I now recall how to recover the local volatility matching an implied volatility surface given on the asset. It is worth noting that, under the hedging assumptions made above, the equation is formally identical to that of a stochastic short rate model having the same diffusion as the instantaneous default probability and therefore, the calibration process follows exactly the same route. The starting point is to write the price of an option using the Green's function, which is the solution to the adjoint equation (the forward Kolmogorov equation):

$$
\begin{aligned}
& \frac{\partial p}{\partial t}-\frac{\partial^{2}}{\partial S^{2}}\left(\frac{1}{2} \sigma^{2} S^{2} p\right)-\frac{\partial^{2}}{\partial \lambda^{2}}\left(\frac{1}{2} a^{2} p\right)-\frac{\partial^{2} V}{\partial \lambda \partial S}(\rho a \sigma S p)+\frac{\partial}{\partial \lambda}(q V)+\frac{\partial}{\partial S}(\lambda S p)+\lambda p=0 \\
& p\left(S_{0}, \lambda_{0}, 0\right)=\delta\left(S-S_{0}, \lambda-\lambda_{0}\right)
\end{aligned}
$$

The price of a contingent option whose payoff depends on $\mathrm{S}$ only is given by integration of its payoff value against the distribution $\mathrm{p}$, that is:

$$
\begin{aligned}
& V\left(S_{0}, \lambda_{0}, 0\right)=\iint \Phi(S) p(S, \lambda, t) d S d \lambda=\int \Phi(S) q(S, t) d S \\
& q(S, t)=\int p(S, \lambda, t) d \lambda
\end{aligned} .
$$


Now, an equation for $\mathrm{q}$ obtains by integrating that for $\mathrm{p}$ with respect to $\lambda$ :

$\frac{\partial q}{\partial t}-\frac{\partial^{2}}{\partial S^{2}}\left(\frac{1}{2} \sigma^{2} S^{2} q\right)+\frac{\partial}{\partial S}\left(S \int \lambda p d \lambda\right)+\int \lambda p d \lambda=0$

$q\left(S_{0}, 0\right)=\delta\left(S-S_{0}\right)$

One now uses the equation for a call option and its connection with $\mathrm{q}$ as in the standard

Dupire formula:

$q(K, T)=\frac{\partial^{2} C}{\partial K^{2}}(K, T)$

and plug it into the equation for q. Upon integrating by parts (note the change of notations to use the standard $(\mathrm{K}, \mathrm{T}))$, one can show that

$\frac{\partial}{\partial T}\left(\frac{\partial^{2} C}{\partial K^{2}}\right)-\frac{\partial^{2}}{\partial K^{2}}\left(\frac{1}{2} \sigma^{2} K^{2} \frac{\partial^{2} C}{\partial K^{2}}\right)-\int \frac{\partial^{2}}{\partial K^{2}}\left(K \int_{K}^{+\infty} p(y, \lambda, t) d y\right) \lambda d \lambda=0$

$p\left(S_{0}, \lambda_{0}, 0\right)=\delta\left(S-S_{0}, \lambda-\lambda_{0}\right)$

which finally yields the forward equation for the call price

$\frac{\partial}{\partial t} C-\frac{1}{2} \sigma^{2} K^{2} \frac{\partial^{2} C}{\partial K^{2}}-\int\left(K \int_{K}^{+\infty} p(y, \lambda, t) d y\right) \lambda d \lambda=0$

and that of the local volatility

$\frac{1}{2} \sigma^{2} K^{2}=\frac{\frac{\partial C}{\partial T}-\int\left(K \int_{K}^{+\infty} p(y, \lambda, t) d y\right) \lambda d \lambda}{\frac{\partial^{2} C}{\partial K^{2}}}$

The calibration issue is therefore naturally cast into a fixed-point problem for the unknown function $\sigma$, its numerical solution problem is best performed using a forward induction method which we will not described in details in this document.

Some comments are in order: firstly, in the case of a deterministic default intensity, one trivially integrates in $\lambda$ to recover the usual formula

$\frac{1}{2} \sigma^{2} K^{2}=\frac{\frac{\partial C}{\partial T}-\bar{\lambda} K \int_{K}^{+\infty} \frac{\partial^{2} C}{\partial K^{2}}(y, T) d y}{\frac{\partial^{2} C}{\partial K^{2}}}=\frac{\frac{\partial C}{\partial T}+\bar{\lambda} K \frac{\partial C}{\partial K}}{\frac{\partial^{2} C}{\partial K^{2}}}$. Secondly, in the case of a non zero,

deterministic risk-free rate, one simply has to replace $\lambda$ by $r+\lambda$, adding an extra term $r K \frac{\partial C}{\partial K}$ to the numerator.

\section{Conclusion}


In this paper, I have presented some real-life models that can be, and actually are, used for the modelling of credit risk in derivatives. After describing the hedging strategy that allows one to obtain a valuation by replication, I have given a simple but useful practical example of joint calibration for a hybrid equity-credit model.

\section{References}

[BJR] T.R. Bielecki, M. Jeanblanc, M. Rutkowski, Hedging of defaultable claims, ParisPrinceton Lectures on Mathematical Finance, Lecture notes in mathematics 1847, p. 1-132, Springer verlag, 2004

[JR] M. Jeanblanc et M. Rutkowski, Modelling and Hedging of credit risk. p.385-416, In Credit derivatives, the definite guide, Application networks, Risk book, 2003.

[S] P. J. Schönbucher, Credit Derivatives Pricing Models: Model, Pricing and Implementation, John Wiley and Sons, 2003

[AB] L. B.G. Andersen, D. Buffum, Calibration and Implementation of Convertible Bond Models, Journal of Computational Finance, vol. 7, part 2, 2004, pages 1-34

[R] W.J. Runggaldier, Jump Diffusion Models, Handbook of Heavy Tailed Distributions in Finance (S.T. Rachev, ed.), Handbooks in Finance, Book 1, W.Ziemba Series, Elsevier/NorthHolland 2003, pp.169-209

[DS] D. Duffie, K. Singleton, Credit risk: pricing, measurement and management, Princeton series in finance, 2003

[DFS] D. Duffie, D. Filipovic, W. Schachermayer, Affine processes and applications in finance, Annals of Applied Probability, vol. 13, n 3, 2003, pp 984-1053

[BBS] H. Beresticki, J. Busca, I. Florent, Computing the implied volatility in stochastic volatility models, Comm. Pure Appl. Math., 57, vol. 10, 2004, p. 1352-1373. 